\title{
Lipid-induced Changes in Intracellular Iron Homeostasis In Vitro and In Vivo
}

\author{
Brian J. Van Lenten, John Prieve, Mahamad Navab, Susan Hama, Aldons J. Lusis, and Alan M. Fogelman \\ Division of Cardiology, Department of Medicine, University of California Los Angeles, Los Angeles, California 90024
}

\begin{abstract}
Iron promotes cellular damage via its capacity to catalyze hydroxyl radical formation and by peroxidation of unsaturated lipids. The major cellular iron storage depot, ferritin, acts as a critical antioxidant defense by sequestering unbound or "free" iron, limiting its participation in damaging oxidative reactions. In this study, we investigated the relationship between LDL modified by artery wall cells and the regulation of intracellular free iron levels in the mouse model and in a human aortic endothelial and smooth muscle cell coculture system. We found in response to an atherogenic diet, fatty streak-resistant $\mathrm{C} 3 \mathrm{H} / \mathrm{HeJ}$ mice exhibited higher levels of liver apoferritin and lower intracellular concentrations of free iron than did fatty streak-susceptible C57 BL/6J mice. Also, ferritin repressor protein mRNA was not significantly suppressed after 15 wk on the atherogenic diet in female C57BL/6J mice, which exhibit the most extensive fatty streak formation, but was significantly reduced in $\mathrm{C} 3 \mathrm{H} / \mathrm{HeJ}$ mice. Iron loading of coculture cells resulted in elevations of cellular free iron and enhanced LDL-induced monocyte transmigration. Pretreatment of cells with apoferritin completely abolished iron-induced LDL modification. Addition of LDL to cocultures resulted in elevations in lipid peroxidation products, intracellular free iron, apoferritin mRNA expression, and apoferritin synthesis, suggesting a possible relationship between the oxidative modification of LDL and iron metabolism. (J. Clin. Invest. 1995. 95:2104-2110.) Key words: atherosclerosis • iron • high fat diet $\bullet$ lipid peroxidation • apoferritin
\end{abstract}

\section{Introduction}

Oxygen free radicals have been implicated in the pathogenic process of a number of diseases such as atherosclerosis (1). Many of the reactions that involve oxygen free radicals often require the presence of a transition metal such as iron (2). Iron can promote cellular damage by way of its capacity to catalyze hydroxyl radical formation and by the peroxidation of unsaturated lipids. Iron in the ferric form is normally bound to proteins, preventing it from participating in reactions that could lead to cellular damage. In the serum, this is accomplished by transferrin, whereas in most cells, iron is bound to apoferritin, a

Address correspondence to Brian J. Van Lenten, Ph.D., Division of Cardiology, Room 47-123, Center for Health Sciences, UCLA School of Medicine, Los Angeles, CA 90024-167917. Phone: 310-206-1150; FAX: 310-825-4963.

Received for publication 16 February 1994 and in revised form 21 December 1994.

J. Clin. Invest.

(C) The American Society for Clinical Investigation, Inc. 0021-9738/95/05/2104/07 \$2.00

Volume 95, May 1995, 2104-2110 multimeric protein consisting of two chains, a light and a heavy chain (3). Hence, intracellular unbound or "free" iron that could catalyze damaging oxidative reactions would be sequestered by ferritin, which would thus act as a critical antioxidant defense for the cell. Studies supporting this concept have come from Balla et al. (4), who demonstrated that apoferritin synthesis was induced in endothelial cells upon exposure to hemin, an iron-containing molecule; this induction resulted in protection against oxidant-mediated cytolysis.

The synthesis of apoferritin appears to be regulated at the translational level by free iron and would therefore be controlled by the intracellular pool of catalytically active iron (3). A prime focus of this regulation is the interaction between a 28-nucleotide sequence in the $5^{\prime}$ untranslated region of apoferritin mRNA, the iron-responsive element or $\operatorname{IRE}^{1}$ (5), and a $90-\mathrm{kD}$ binding protein known as IRE-binding protein or ferritin repressor protein (FRP) (6). Normally, the cellular pool of ferrous iron is small, which favors the binding of FRP to the IRE and hence the inhibition of apoferritin mRNA translation. However, when there is an increase in the ferrous iron pool, the association of the IRE with FRP is disrupted, and the translation of apoferritin mRNA can proceed. Recent data, however, from Coulson and Cleveland (7) require an expanded interpretation of the interaction between intracellular iron and the FRP-IRE complex. In their studies, heavy chain ferritin mRNA levels, but not stability, were altered by elevating the level of intracellular iron. They concluded that iron-dependent control of ferritin synthesis is regulated by a combination of translational control and regulation of ferritin mRNA abundance. Thus, mechanisms beyond IRE-dependent translational control contribute significantly to regulation of ferritin synthetic rates.

Based on in vitro studies of endothelial cell and smooth muscle cell cocultures (8), as well as from in vivo studies (9, 10 ), we have postulated that mild oxidation of LDL by lipid peroxides generated by artery wall cells and transferred to LDL in a microenvironment protected from antioxidants produces a minimally modified LDL, or MM-LDL. This particle may then initiate the inflammatory process that ultimately leads to the formation of the atherosclerotic lesion. It is known that the mouse strains $\mathrm{C} 57 \mathrm{BL} / 6 \mathrm{~J}$ and $\mathrm{C} 3 \mathrm{H} / \mathrm{HeJ}$ differ in their susceptibilities to fatty streak formation and that female mice are more susceptible to fatty streak formation than male mice (11). In the present study, we found that in response to an atherogenic diet, fatty streak-resistant mice, $\mathrm{C} 3 \mathrm{H} / \mathrm{HeJ}$, exhibited higher levels of liver apoferritin and lower intracellular concentrations of free iron than did the fatty streak susceptible strain C57 BL/ 6J. Moreover, FRP mRNA was not significantly suppressed after $15 \mathrm{wk}$ on the atherogenic diet in the mice exhibiting the most extensive fatty streak formation, female C57BL/6J, but

1. Abbreviations used in this paper: FRP, ferritin repressor protein; HAEC, human aortic endothelial cell; HASML, human aortic smooth muscle cell; HO, heme oxygenase; 8-HQ, 8-hydroxyquinoline; IRE, iron-responsive element; MM-LDL, minimally oxidized LDL; NO, nitric oxide. 
was significantly reduced in the other mice. The modification of LDL by endothelial cell/smooth muscle cell cocultures was enhanced by iron loading of the cells, which could be counteracted by apoferritin. In vitro, the production of MM-LDL by cocultures resulted in an increase in intracellular free iron as well as increases in both apoferritin mRNA expression and apoferritin synthesis. These data suggest that lipid peroxidation may be an important variable in intracellular iron homeostasis.

\section{Methods}

\section{Mice}

20 male and 20 female C57 BL/6J and C3H/HeJ mice, 2-3 mo of age, were purchased from the Jackson Laboratory (Bar Harbor, ME). Animals were divided into groups of five mice each and fed either a normal Purina chow diet (Ralston Purina Co., St. Louis, MO) or an atherogenic diet of $15 \%$ fat, $1.25 \%$ cholesterol, and $0.5 \%$ cholic acid (Teklad, Madison, WI) for $15 \mathrm{wk}$. At the end of this period, the mice were anesthetized with Forane (Ohmeda, Madison, WI) and bled from the orbital sinus for the measurement of apoferritin and transferrin levels in the plasma. The mice were then perfused with $0.15 \% \mathrm{NaCl}$ via the portal vein to clear the tissue of blood and were sacrificed. The hearts and livers were removed and transferred to liquid nitrogen for histological assessment of fatty streak development and the quantitation of specific mRNA and proteins, respectively.

\section{Histological analysis}

The heart, including the aortic root, was dissected out and washed once in PBS. The basal portion of the heart and aortic root were embedded in OCT compound (Miles Inc., Elkhart, IN) and frozen on dry ice. Serial $10-\mu$ m-thick cryosections of the heart tissue, covering the area between the appearance of the mitral valve to the disappearance of the aortic valve, were prepared. Every third section was collected on a poly-L-lysinecoated slide. All sections were stained with oil Red O (Sigma Chemical Co., St. Louis, MO) to assess aortic fatty streak development. Quantitation of lipid deposition was conducted as previously described (11).

\section{Western blot analysis}

To quantitate the concentrations of mouse liver apoferritin, tissue samples were first homogenized in PBS containing $1 \%$ SDS using a polytron, and unsolubilized material was then removed by centrifugation at $10,000 \mathrm{~g}$. The supernatants were assayed for protein content using a kit for assaying protein in the presence of detergents (DC kit; Bio Rad, Richmond, CA). Equal amounts of supernatant protein (100 ng) were loaded into wells for slot-blot analysis along with a set of purified apoferritin standards (Sigma Chemical Co.) and applied to a nitrocellulose membrane (Hybond ECL; Amersham Corp., Arlington Heights, IL) in Tris-buffered saline. Chemiluminescent detection of proteins was performed using an ECL Western blotting kit (Amersham Corp.) according to the protocol supplied with the kit. The primary antibody used was rabbit anti-human ferritin (Boehringer Biochemicals, Mannheim, Indianapolis, IN), which also showed high cross-reactivity with mouse liver apoferritin. The antiserum was shown to give only one precipitin line in crossed immunoelectrophoresis using a ratio of $6 \mu \mathrm{l}$ of antiserum per $\mathrm{cm}^{2}$ to $1 \mu \mathrm{l}$ of lysate. The secondary antibody used was horseradish peroxidase-conjugated anti-rabbit IgG (Amersham Corp.). The blots were exposed to Hybond ECL film and densitometrically scanned for quantitation.

\section{Quantitation of gene expression}

Total RNA was extracted from mouse liver samples as well as from the cocultures using the technique of Chomczynski and Sacchi (12). Northern blot analysis was used to quantitate the mRNA levels of FRP in mouse liver and the mRNA for the heavy chain of apoferritin in coculture cells. For the determination of FRP expression, RNA samples were diluted with equal volumes of SSC/formaldehyde (60\% 20× SSC and $40 \%$ formaldehyde), incubated at $65^{\circ} \mathrm{C}$, and then cooled to room temperature before sample loading. $1 \mu \mathrm{g}$ of RNA was loaded in each well and transferred to $20 \times$ SSC equilibrated Hybond ECL nitrocellulose membrane by using a dot-blot chamber. Films were hybridized after UV cross-linking and washed at a high stringency $\left(65^{\circ} \mathrm{C}, 0.1 \times\right.$ $\mathrm{SSC}$ ). The murine FRP probe had the sequence 5'-TTGTCGGAGGTGCTTGGTAATGGTGAG-3'. The blots were also probed with murine $\beta$-actin to normalize the quantities of RNA loaded into the gel lanes. For the quantitation of apoferritin mRNA in coculture cells, Northern blot analysis was performed as described (13) using a probe with the sequence 5'-CTGGCGCACCTGCGAGGTGGAGCGGGTCG-3' and a cDNA probe for $\alpha$-tubulin to normalize the quantities of RNA loaded into the gel lanes.

\section{Cocultures}

Human aortic endothelial cells (HAEC) and smooth muscle cells (HASMC) were isolated as previously described $(8,14)$. Transwells or Chamber slides were used for formation of cocultures and for the study of monocyte transmigration. The wells were treated with $0.1-$ $0.5 \%$ gelatin at $37^{\circ} \mathrm{C}$ overnight. HASMC were seeded in units or on membranes at a confluent density of $10^{5}$ cells per $\mathrm{cm}^{2}$ and were cultured for $3 \mathrm{~d}$, at which time they had covered the entire surface of the well and had produced a substantial amount of extracellular matrix. The HASMC surface was treated with $20 \mu \mathrm{g} / \mathrm{ml}$ human fibronectin in medium 199 for $10 \mathrm{~min}$, which was removed before seeding with HAEC. HAEC were subsequently seeded at $2 \times 10^{5}$ cells per $\mathrm{cm}^{2}$ in medium 199 containing $20 \%$ pooled human serum and FBS (1:1) and were allowed to grow, forming a complete monolayer of confluent endothelial cells in $2 \mathrm{~d}$. In all experiments, HAEC and autologous HASMC (from the same donor) were used at the fifth to sixth passage. Identical results were obtained with multilayer cocultures produced in the different systems (i.e., Transwells or Chamber slides). Blood monocytes were obtained from a large pool of healthy donors by modification of the Recalde procedure as previously described (15). Cocultures in medium 199 containing $2 \%$ pooled human serum were treated with native LDL ( 350 $\mu \mathrm{g} / \mathrm{ml}$ ) in the absence or presence of various test compounds for $48 \mathrm{~h}$.

Iron loading of cocultures. Iron loading of the cocultures was performed according to Balla et al. (4), using the iron-chelating compound 8-hydroxyquinoline (8-HQ). Loading was accomplished by first incubating coculture cells in medium 199 with $10 \mu \mathrm{M} \mathrm{FeSO}{ }_{4} \cdot 7 \mathrm{H}_{2} \mathrm{O}$ and 10 $\mu \mathrm{M} 8-\mathrm{HQ}$ for $30 \mathrm{~min}$. Cells were then washed three times with PBS, followed by the addition of test substances. Some cocultures were pretreated with apoferritin for 1-2 h before incubation with iron and 8$\mathrm{HQ}$. There was a 2.7-fold increase in the free iron content of the coculture cells after iron loading using $10 \mu \mathrm{M}$ iron and $10 \mu \mathrm{M}$ 8- $\mathrm{HQ}$ (from $2.4 \pm 0.01$ to $6.5 \pm 0.03 \mathrm{nmol}$ of iron per $10^{6}$ cells). Cytotoxicity was evaluated using propidium iodide as a fluorescent probe, which was added to cultures at a final concentration of $50 \mu \mathrm{M}$. Fluorescence at $536 \mathrm{~nm}$ excitation and $590 \mathrm{~nm}$ emission $\left(f_{1}\right)$ was determined on a Cytofluor 2300 (Millipore, Bedford, MA). Digitonin at $200 \mu \mathrm{M}$ was subsequently added, and fluorescence was determined $\left(f_{2}\right)$. The ratio $f_{1} / f_{2}$ was used to determine the level of cytotoxicity. Exposure of cells for $30 \mathrm{~min}$ to $10 \mu \mathrm{M}$ concentrations of both 8-HQ and iron, the concentrations used in these studies, was not toxic. Under the same iron-loading conditions, using trypan blue exclusion, cells were found to be $>95 \%$ viable. Chelation of extracellular iron in the culture medium was performed using the iron chelator DETAPAC (Sigma Chemical Co.).

Free iron content of cocultures. The free iron content of cells in cocultures was estimated using bleomycin-dependent degradation of DNA as previously reported (16), which detects iron bound to compounds with a lower affinity for iron than bleomycin. Briefly, after incubation of cocultures with iron and 8-HQ, the cells were first washed in medium 199, followed by two washes with PBS, and then pelleted at $200 \mathrm{~g}$ for $10 \mathrm{~min}$. The cell pellet was lysed in $500 \mu \mathrm{l}$ of a buffer containing $50 \mathrm{mM} \mathrm{NaCl}, 20 \mathrm{mM}$ Tris, $\mathrm{pH} 7.5$, and $0.1 \%$ Triton X-100. After a 15-min incubation at room temperature, the cell lysate was centrifuged at $14,000 \mathrm{~g}$ for $5 \mathrm{~min}$, and $100-\mu \mathrm{l}$ aliquots of the supernatant in triplicate were used for analysis of free iron.

Metabolic labeling of cells. To measure the extent of apoferritin synthesis in cocultures, metabolic labeling of cells was performed as previously described (14). Cells were labeled with $\left[{ }^{35} S\right]$ methionine for 


\begin{tabular}{|c|c|c|c|c|c|c|}
\hline Strain & Sex & Diet & Lesion area & Free iron & Apoferritin & Free iron/apoferritin \\
\hline & & & $\mu m^{2} /$ section & nmol/mg & $p g / m g$ & \\
\hline C3H/HeJ & Male & Control & 0 & $149 \pm 25$ & $113 \pm 4$ & $1.31 \pm 0.13$ \\
\hline C3H/HeJ & Female & Control & 0 & $120 \pm 2$ & $125 \pm 12$ & $0.96 \pm 0.05$ \\
\hline C57BL/6J & Male & Control & 0 & $90 \pm 5$ & $79 \pm 14$ & $1.13 \pm 0.13$ \\
\hline C57BL/6J & Female & Control & 0 & $93 \pm 9$ & $136 \pm 11$ & $0.68 \pm 0.05$ \\
\hline С3H/HeJ & Male & HF & 0 & $57 \pm 28 *$ & $145 \pm 12 *$ & $0.40 \pm 0.11^{*}$ \\
\hline $\mathrm{C} 3 \mathrm{H} / \mathrm{HeJ}$ & Female & HF & $622 \pm 179 *$ & $78 \pm 9 *$ & $155 \pm 12^{*}$ & $0.51 \pm 0.05^{*}$ \\
\hline C57BL/6J & Male & HF & $1,000 \pm 330^{*}$ & $69 \pm 18$ & $51 \pm 3$ & $1.34 \pm 0.21$ \\
\hline C57BL/6J & Female & HF & $12,291 \pm 2,701^{*}$ & $86 \pm 9$ & $112 \pm 18$ & $0.77 \pm 0.14$ \\
\hline
\end{tabular}

Male and female $\mathrm{C} 3 \mathrm{H} / \mathrm{HeJ}$ and C57BL/6J mice were fed either normal control chow or an atherogenic diet $(H F)$ for 15 wk. At the end of this time, hearts and livers were removed for histological assessment of fatty streak development and the quantitation of hepatic free iron and apoferritin, respectively, as described in Methods. Values are presented as the mean \pm SD of five animals. ${ }^{*}$ Statistical difference from control at $P<0.05$.

$2 \mathrm{~h}$ in methionine-free medium, medium was collected, and protease inhibitors were added. The cultures were then washed with medium containing $40 \mu \mathrm{M}$ nonradioactive methionine and incubated again for $2 \mathrm{~h}$. The chase medium was collected, protease inhibitors were added, the medium was centrifuged at $350 \mathrm{~g}$, and the supernatants were collected and stored at $-20^{\circ} \mathrm{C}$.

Immunoprecipitation of radiolabeled apoferritin. Before immunoprecipitation, the thawed media were precleared with protein A-Sepharose (Sigma Chemical Co.). TCA-precipitable counts were determined. To equal TCA-precipitable counts from each treatment sample (50$200 \mu \mathrm{l}$ ) was added $10 \mu \mathrm{l}$ of rabbit anti-human ferritin (Boehringer Biochemicals), and the volume was adjusted to $500 \mu \mathrm{l}$ with medium. After polyacrylamide gel electrophoresis of the samples, gels were fixed, treated with Enhance (DuPont/NEN, Boston, MA), and autoradiographed. Densitometric scanning was performed using the Image 1.51 program (NIH).

\section{Other procedures}

LDL ( $d=1.019-1.063 \mathrm{~g} / \mathrm{ml})$ were isolated from the sera of normal blood donors by density gradient ultracentrifugation as described (17) and were used within 1 wk of isolation. Measurement of cell protein content and of lipoproteins was by the method of Lowry et al. (18). Measurement of lipid hydroperoxides was performed according to the method of Auerbach et al. (19).

Statistical analyses were performed using Model I ANOVA to determine whether differences existed among the group means, followed by a paired Student's $t$ distribution to identify the significantly different means.

\section{Results}

Genetic variations in iron homeostasis. Consistent with previous observations (11), only C57BL/6J females exhibited a dramatic increase in fatty streak formation after $15 \mathrm{wk}$ on the atherogenic diet (Table I). To determine whether differences in iron homeostasis exist between the two strains of mice that could contribute to their differences in lesion development, the free iron and apoferritin contents of livers from both male and female mice were quantitated (Table I). Feeding the atherogenic diet resulted in similar profiles for both sexes, that is, decreases in the free iron content and increases in liver apoferritin levels in $\mathrm{C} 3 \mathrm{H} / \mathrm{HeJ}$ but not in C57BL/6J. If these data are viewed as a ratio of the free iron value to the apoferritin value, there is an even more pronounced difference between the two strains: $\mathrm{C} 3 \mathrm{H} / \mathrm{HeJ}$ mice showed a marked decrease, whereas C57BL/6J mice exhibited a trend toward an increase in the free iron/apoferritin ratio. These results suggest that in contrast to C57BL/6J mice, feeding the atherogenic diet to $\mathrm{C} 3 \mathrm{H} / \mathrm{HeJ}$ mice either directly or indirectly resulted in an increase in the liver apoferritin protein content, which in turn reduced cellular free iron levels.

Since apoferritin synthesis is regulated to a high degree by the activity of a repressor protein, FRP, mRNA levels of liver FRP were measured as shown in Fig. 1. After 15 wk on the atherogenic diet, only the C57 females, the group demonstrating the greatest fatty streak development on the atherogenic diet, did not show a statistically significant decrease in FRP mRNA expression. In contrast, neither plasma apoferritin nor plasma transferrin levels were different among the groups after feeding the atherogenic diet (Table II).

Iron promotes $L D L$ modification in vitro. Modification of LDL in serum-containing multilayer cultures of artery wall cells to form MM-LDL induces increased monocyte adhesion to and transmigration through the endothelial cell layer in the cocultures (20). We use monocyte transmigration as a biologic mea-

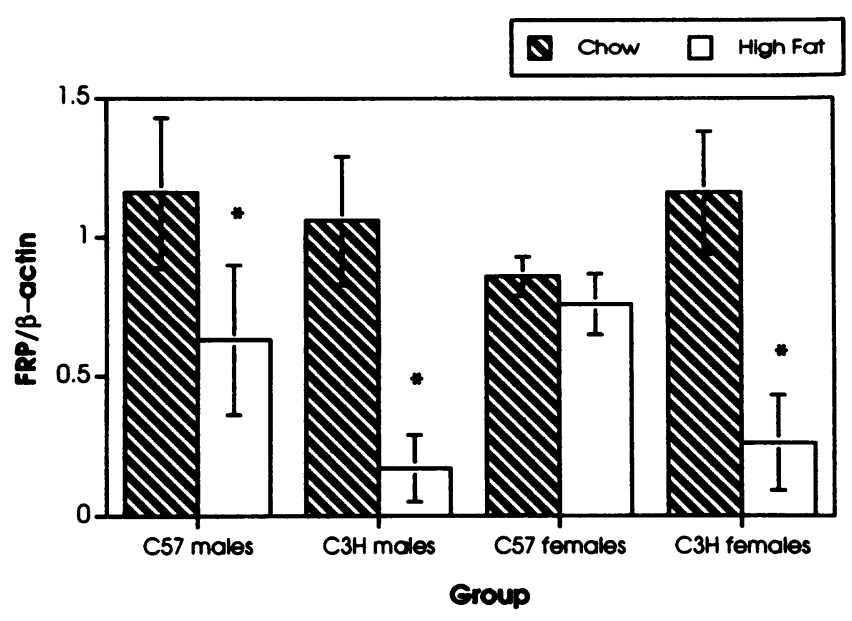

Figure 1. Hepatic FRP mRNA levels in male and female $\mathrm{C} 3 \mathrm{H} / \mathrm{HeJ}$ and C57BL/6J mice. After 15 wk on either normal chow or an atherogenic diet, liver RNA was prepared as described in Methods and the content of FRP was determined from Northern blot analysis. Values are presented as the mean $\pm S D$ of five animals. Asterisks indicate a statistically significant difference from chow-fed controls at $P<0.05$. 
Table II. Plasma Apoferritin and Transferrin Levels in $\mathrm{C} 3 \mathrm{H} / \mathrm{HeJ}$ and C57BL/6J Mice

\begin{tabular}{|c|c|c|c|c|}
\hline Strain & Sex & Diet & Transferrin & Ferritin \\
\hline & & & $m g / \mu g$ protein & $m g / \mu g$ protein \\
\hline $\mathrm{C} 3 \mathrm{H} / \mathrm{HeJ}$ & Male & Control & $1.13 \pm 0.24$ & $1.04 \pm 0.33$ \\
\hline $\mathrm{C} 3 \mathrm{H} / \mathrm{HeJ}$ & Female & Control & $1.28 \pm 0.17$ & $0.84 \pm 0.23$ \\
\hline C57BL/6J & Male & Control & $1.12 \pm 0.11$ & $1.25 \pm 0.55$ \\
\hline C57BL/6J & Female & Control & $0.90 \pm 0.27$ & $1.37 \pm 0.85$ \\
\hline $\mathrm{C} 3 \mathrm{H} / \mathrm{HeJ}$ & Male & HF & $1.26 \pm 0.27$ & $0.77 \pm 0.16$ \\
\hline C3H/HeJ & Female & HF & $1.70 \pm 0.27$ & $0.89 \pm 0.19$ \\
\hline C57BL/6J & Male & HF & $1.35 \pm 0.29$ & $1.34 \pm 0.61$ \\
\hline C57BL/6J & Female & HF & $1.59 \pm 0.33$ & $1.09 \pm 0.25$ \\
\hline
\end{tabular}

After 15 wk on either normal control chow or an atherogenic diet $(H F)$, plasma apoferritin and transferrin levels in male and female $\mathrm{C} 3 \mathrm{H} / \mathrm{HeJ}$ and C57BL/6J mice were determined as described in Methods. Values are presented as the mean \pm SD of five animals.

sure of LDL modification (oxidation). Whereas cells incubated in the absence of LDL contained no lipid peroxidation products, incubation of cocultures with LDL resulted in lipid peroxidation $(2.08 \pm 0.08 \mathrm{nmol}$ of linoleate used per $10 \mathrm{~min})$. Since iron loading of endothelial cells has been shown to increase their ability to generate lipid peroxides (21), we tested the effects of increased levels of free iron on LDL modification by the cocultures. We found that increased concentrations of iron in the medium, ranging from 0.2 to $0.7 \mu \mathrm{M}$ during $48 \mathrm{~h}$ of incubation, resulted in a progressive increase in LDL modification and the resulting monocyte transmigration (Fig. $2 A$ ). In addition, as shown in Fig. $2 B$, rapid iron loading of the artery wall cells by a 30-min incubation with $10 \mu \mathrm{M}$ iron together with the iron chelator $8-\mathrm{HQ}$ at $10^{-7}-10^{-5} \mathrm{M}$ in the presence of LDL increased monocyte transmigration by up to twofold $(P<0.02$ for $10^{-5} \mathrm{M} 8$-HQ). Moreover, there was a 2.7 -fold increase in the free iron content of the coculture cells after iron loading in the presence of LDL using $10 \mu \mathrm{M}$ iron and $10 \mu \mathrm{M} 8-\mathrm{HQ}$ (from $2.4 \pm 0.01$ to $6.5 \pm 0.03 \mathrm{nmol}$ of iron per $10^{6}$ cells). Pretreatment of cocultures with apoferritin and subsequently treatment with 8-HQ at $10^{-5} \mathrm{M}$ completely prevented the increased monocyte transmigration $(P<0.012)$. This effect of preloading with apoferritin suggests that increased intracellular free iron is responsible for the enhanced LDL-induced monocyte chemotactic activity.

In data not shown, iron loading with higher concentrations of 8-HQ $\left(10^{-3} \mathrm{M}\right)$ resulted in cytotoxicity in the first set of cocultures, as measured by propidium iodide and the absence of monocyte transmigration in the target cocultures. Also, we found that pretreatment with apoferritin of cocultures that were subsequently treated with $10^{-3}$ M 8-HQ prevented the cytotoxicity and resulted in a monocyte transmigration level similar to that observed in the presence of apoferritin and $10^{-5} \mathrm{M} 8-\mathrm{HQ}$ (data not shown).

The in vitro modification of LDL by the use of high concentrations of transition metals has been used by a number of investigators $(22,23)$. Their results demonstrate that the presence of cells is not a requirement for the metal-catalyzed oxidative modification of LDL. We conducted an experiment using apoferritin and 8-HQ in a cell-free system and observed that, similar to the results shown in Fig. 2 measuring monocyte transmigration, LDL incubated in the presence of 8 -HQ and iron
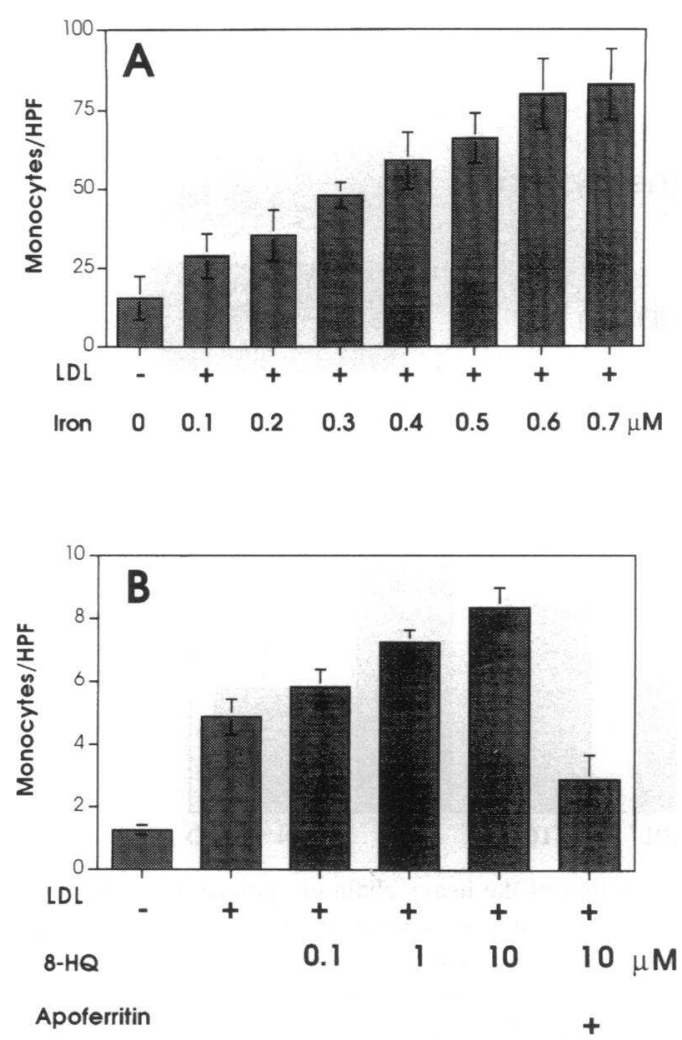

Figure 2. (A) Effect of medium iron content on LDL modification by endothelial cell/smooth muscle cell cocultures. Cocultures were incubated in medium containing either no additional iron $(0)$ or LDL (-), or with an additional $0.1-0.7 \mu \mathrm{M}$ iron and LDL at $250 \mu \mathrm{g} / \mathrm{ml}$ for 24 $\mathrm{h}(+)$. Conditioned medium was then transferred to target cocultures and was further incubated for $24 \mathrm{~h}$. The medium was removed after 24 $h$ of incubation, and a suspension of Dil-labeled monocytes was added at $2.5 \times 10^{5}$ cells per $\mathrm{cm}^{2}$ to the endothelial side of the cocultures. The cultures were returned to the incubator and maintained for 45-90 min. The medium was then removed, and cultures were washed and fixed with $10 \%$ neutral buffered formalin for $24 \mathrm{~h}$. The cocultures were then mounted, and subendothelial monocytes were enumerated under $\times 625$. The values shown are the mean $\pm S D$ in 15 fields from triplicate cocultures. This figure is representative of three independent experiments. $(B)$ Effect of iron loading. Cocultures were incubated in medium containing $10 \mu \mathrm{M}$ iron and the indicated levels of the iron chelator 8-HQ for $30 \mathrm{~min}$. The cells were subsequently washed. Some cocultures were pretreated with $2.0 \mathrm{mg} / \mathrm{ml}$ apoferritin for $2 \mathrm{~h}$ and were thoroughly washed before treatment with iron and 8-HQ. The cultures were incubated with $\mathrm{LDL}$ at $250 \mu \mathrm{g} / \mathrm{ml}$ for $24 \mathrm{~h}$, and conditioned medium was then transferred to target cocultures and further incubated for $24 \mathrm{~h}$. The monocyte transmigration assay was conducted as described for $A$. The values shown are mean $\pm S D$ in 15 fields from triplicate cocultures. This figure is representative of three independent experiments.

resulted in an increase in lipid hydroperoxide production that could be prevented by the addition of apoferritin (no LDL, $0.32 \pm 0.09 \mathrm{nmol} / 10 \mathrm{~min}$; LDL alone, $0.35 \pm 0.11 \mathrm{nmol} / 10 \mathrm{~min}$; $\mathrm{LDL}+8-\mathrm{HQ}+$ iron, $11.93 \pm 0.34 \mathrm{nmol} / 10 \mathrm{~min} ; \mathrm{LDL}+$ apoferritin + 8-HQ + iron, $1.49 \pm 0.43 \mathrm{nmol} / 10 \mathrm{~min}$.) Although these data suggest a possible role for intracellular alterations in iron content in lipid peroxidation, one cannot exclude the potential contribution of extracellular events to the modification of LDL.

Modified LDL influences apoferritin expression. Our laboratory has previously shown that in mice, an atherogenic dietary challenge as well as an injection of MM-LDL increases the 


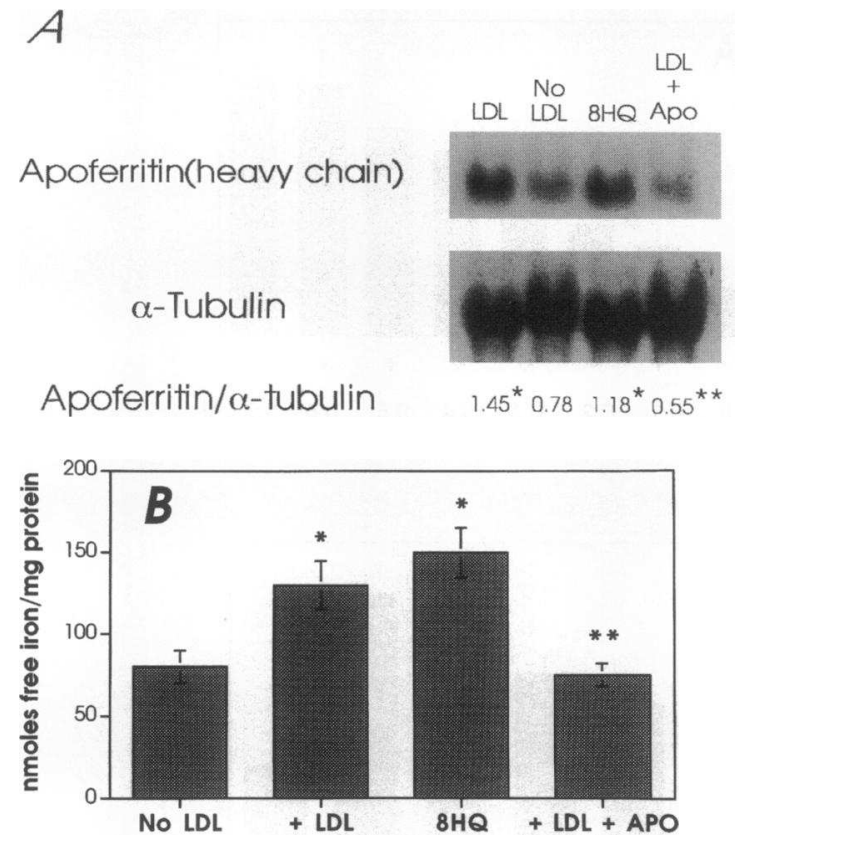

Figure 3. (A) Induction of the heavy chain of apoferritin mRNA by LDL in cocultures. Cocultures were incubated in medium containing either no additional LDL (No $L D L), 350 \mu \mathrm{g} / \mathrm{ml}$ LDL ( $L D L)$, or 10 $\mu \mathrm{M}$ each of iron and the iron chelator $8-H Q(8-H Q)$, or were pretreated with $2.0 \mathrm{mg} / \mathrm{ml}$ apoferritin for $2 \mathrm{~h}$, subsequently washed, and then incubated with $350 \mu \mathrm{g} / \mathrm{ml} \mathrm{LDL}(L D L+A p o)$ for $48 \mathrm{~h}$. RNA was prepared for Northern blot analysis as described in Methods. Expression of $\alpha$-tubulin mRNA is shown to allow normalization of the quantities of RNA loaded in gel lanes (Apoferritin/ $\alpha$-tubulin). Asterisks represent a statistically significant difference $(P<0.05)$ from $N o L D L\left(^{*}\right)$ or from $L D L(* *)$. This figure is representative of three independent experiments. (B) Free iron content of coculture cells. Cocultures treated as in $A$ were prepared for free iron analysis as described in Methods. Values are presented as the mean \pm SD of triplicate determinations and are representative of three independent experiments. Asterisks represent a statistically significant difference $(P<0.05)$ from No $L D L\left(^{*}\right)$ or from $+L D L(* *)$.

expression of inflammatory and oxidative stress responsive genes in the liver (9). Likewise, MM-LDL produced in vitro by a coculture of aortic endothelial and smooth muscle cells induces cytokine synthesis (8). To determine whether MMLDL generated by the arterial coculture system alters apoferritin mRNA expression or protein synthesis, the experiments shown in Fig. 3 were performed. Compared with coculture cells incubated without additional LDL in the tissue culture medium, a 48-h exposure of coculture cells to LDL resulted in an increase in the expression of the mRNA for the heavy chain of ferritin similar to the extent seen when cells were treated with the ironchelating compound 8-HQ (Fig. $3 \mathrm{~A}$ ). If the cells were first pretreated with apoferritin for $2 \mathrm{~h}$, washed, and then exposed to LDL for $48 \mathrm{~h}$, this increase in apoferritin mRNA was not seen. Corresponding with these results (Fig. $3 \mathrm{~B}$ ), the level of free iron in the coculture cells increased in LDL-treated cells similar to that seen with the 8-HQ-positive control for iron loading. Apoferritin pretreatment of the cells suppressed the LDL-induced increase in free iron. Consistent with the changes observed in apoferritin mRNA, $\left[{ }^{35} \mathrm{~S}\right]$ methionine incorporation into coculture cell apoferritin was greater after LDL treatment and was inhibited by apoferritin pretreatment (Fig. 4). These alterations in mRNA expression and synthesis of apoferritin

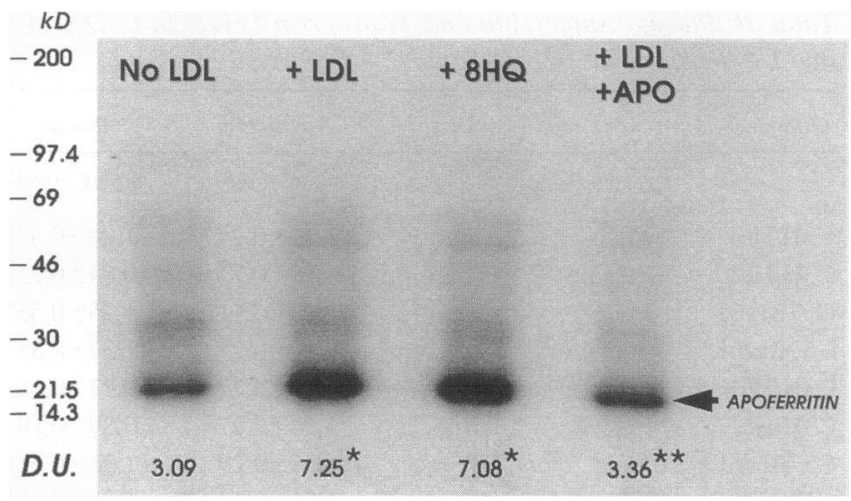

Figure 4. Apoferritin synthesis induced by LDL in cocultures. Cocultures were treated as in Fig. 3, and the incorporation of ${ }^{35} \mathrm{~S}$ into apoferritin was performed as described in Methods. Equal TCA-precipitable counts in the cell lysates were loaded in each lane. The autoradiogram shown is representative of three experiments. Molecular weight protein markers are shown on the left. Densitometry units (D.U.) are defined as the optical density of the band per $\mathrm{mm}^{2}$ using Image 1.51. Asterisks represent a statistically significant difference $(P<0.05)$ from No $L D L$ $\left(^{*}\right)$ or from $+L D L(* *)$.

were also associated with changes in the generation of lipid hydroperoxides. Under these same experimental conditions, the addition of LDL to cocultures increased the generation of lipid hydroperoxides from $0.06 \pm 0.09$ to $1.00 \pm 0.09 \mathrm{nmol}$ of linoleate used per $10 \mathrm{~min}$. Pretreatment of cocultures with apoferritin before addition of LDL suppressed the LDL-induced increase in lipid hydroperoxides $(0.43 \pm 0.04 \mathrm{nmol}$ of linoleate used per $10 \mathrm{~min}$ ).

To determine whether the observed results were changes in the intracellular iron status, cells were preloaded with iron, washed, and then incubated with LDL alone or in the presence of the iron chelator DETAPAC at a dose $(0.1 \mathrm{M})$ known to chelate all effective extracellular iron (24). There were no significant differences in the number of monocytes transmigrated per high powered field (no additions, $2.42 \pm 0.79$; + LDL alone, 8.08 \pm 1.08 ; + LDL + DETAPAC, 7.83 \pm 1.07 ). Likewise, coincubation of cocultures with DETAPAC failed to decrease the LDL-induced generation of lipid hydroperoxides $(2.37 \pm 0.14$ vs $2.48 \pm 0.19 \mathrm{nmol}$ of linoleate used per $10 \mathrm{~min}$, respectively). This suggests that alterations in the intracellular iron status of the cell may play a role in lipoprotein modification.

\section{Discussion}

There is a growing body of evidence that oxidative modification of LDL may play a significant role in the development of atherosclerotic lesions. In mice, observed differences in the susceptibilities of various strains to fatty streak formation may be due in part to differences in how the animals respond to oxidative challenges. Liao et al. (25) have recently provided strong evidence for the role of inflammatory mediators inducible by oxidative stress in atherogenesis. Induction of inflammatory genes and activation of NF- $\kappa$ B-like transcription factors were shown to cosegregate with aortic fatty streaks in mice.

By virtue of its reactivity with activated oxygen, iron can promote the formation of oxidant species such as hydroxyl radicals, which contribute to lipid peroxidation. Sequestration of cellular iron in the iron storage protein ferritin can limit the 
participation of the highly reactive free iron in these oxidative reactions. Our results demonstrate that the fatty streak-susceptible mouse strain, C57BL/6J, did not maintain iron homeostasis as well as the fatty streak-resistant strain, $\mathrm{C} 3 \mathrm{H} / \mathrm{HeJ}$, when fed an atherogenic diet. The $\mathrm{C} 3 \mathrm{H} / \mathrm{HeJ}$ strain on the atherogenic diet exhibited decreases in free iron accumulation and in FRP message expression and an increase in liver apoferritin levels. In contrast, the fatty streak-susceptible strain, C57BL/6J, was significantly less efficient at maintaining iron balance in the face of a lipid-induced oxidative stress. The synthesis of apoferritin (as well as the transferrin receptor) is regulated by an IRE-FRP interaction, such that an increase in cellular free iron acts to disrupt this interaction, removing the repression by FRP and thus allowing apoferritin synthesis to proceed. In this study, the expression of FRP message appeared to correlate with the observed differences in fatty streak development in the mice: the mice with the greatest lesion incidence, female C57BL/6J, also showed no significant decrease in FRP message, consistent with the attenuated apoferritin and free iron responses seen in these mice (Table I and Fig. 1).

Our data obtained from human aortic cell cocultures support a role for apoferritin as a cytoprotectant against oxidative damage. Our previous work has shown that incubation of LDL with endothelial/smooth muscle cell cocultures results in the generation of MM-LDL, a particle capable of inducing increased adhesion and transmigration of monocytes (8). Our present study demonstrated that a 48-h incubation of coculture cells with LDL results in an increase in the cellular free iron content, an increase in the expression of the mRNA for the heavy chain of ferritin, as well as an increase in the synthesis of apoferritin protein (Figs. 3 and 4). Juckett et al. (22) have recently provided evidence that apoferritin can protect endothelial cells from hemin-oxidized LDL-mediated cytotoxicity and that this protection resides in the heavy chain of ferritin. Since it is known that the heavy chain of ferritin contains ferroxidase activity, this increased synthesis of apoferritin may confer a resistance to redox reactions that would act to propagate oxidative damage (26). Other investigators have shown an ironinduced increase in apoferritin transcription. Coccia et al. (27) have shown that in erythroleukemic cells, exposure to hemin leads to a 10-fold increase in ferritin mRNA levels, with a 20fold increase in ferritin protein expression. Liau et al. (28) demonstrated an elevation in ferritin heavy chain mRNA expression in rabbit smooth muscle cells after treatment with hemin. Our results suggest that the production of MM-LDL by coculture cells results in an increase in the intracellular free iron content, which in turn results in an increase in apoferritin synthesis.

Effects of MM-LDL on FRP may require days or weeks to become expressed. In the fatty streak-resistant mouse, $\mathrm{C} 3 \mathrm{H} /$ HeJ, decreases in FRP were observed after 15 wk when on the atherogenic diet. However, we did not see any differences in FRP mRNA expression in the coculture system after $48 \mathrm{~h}$ of incubation with LDL (data not shown). One would expect that before changes in FRP synthesis are observed, changes in the binding activity of FRP with the IRE may occur (29). Initially, such a posttranslational modification could be the first line of defense against changes in iron status. One might also reason that with a prolonged oxidative challenge, such as that presented by a 15-wk atherogenic diet, the need for dealing with an increased accumulation of intracellular free iron would be greater. This would require increases (possibly at the transcriptional level) in the ferritin synthetic machinery necessary to sequester reactive iron and maintain iron homeostasis (7).

While the data on free iron and apoferritin levels are consistent with the differing susceptibilities between C57BL/6J and $\mathrm{C} 3 \mathrm{H} / \mathrm{HeJ}$ mice to fatty streak formation, they do not adequately explain the marked difference in susceptibilities between males and females since the male C57BL/6J mice demonstrated iron/ apoferritin ratios similar to those of females but had much less fatty streak formation (Table I). Only the mRNA levels of FRP clearly differentiated the C57BL/6J female mice from the other groups (Fig. 1). Contrary to the data of Salonen et al. (30) in humans, plasma levels of ferritin did not differentiate between fatty streak-susceptible and-resistant animals (Table II). Our data suggest that genetic differences in iron metabolism in response to an atherogenic diet exist and may be related to lesion development. However, the differing susceptibilities of male and female mice to lesion formation are not a simple function of iron status, but are likely due to a complex interaction between multiple factors of which iron status is potentially but one.

The indirect effects of lipid peroxidation products on iron metabolism should also be considered. One possible scenario is suggested by the work of Liao et al. (10), who demonstrated that feeding mice an atherogenic diet increased the expression of heme oxygenase (HO) mRNA concomitant with an increase in lipid peroxidation products. The induction of $\mathrm{HO}$ by lipid peroxidation products would be expected to increase the degradation of heme released from damaged cellular heme proteins. This would in turn generate increased levels of heme-derived iron, which could act as critical catalysts in oxidant-induced damage. Recent studies from Nutter et al. (31) support the idea that induction of $\mathrm{HO}$, rather than being a protective response against oxidant stress, could in fact potentiate cellular damage.

Another line of evidence suggests that inflammation can initiate changes in ferritin homeostasis independently of changes in cellular iron concentrations. TNF- $\alpha$ (32) and IL-1$\beta$ (33), two cytokines released in response to inflammation, have been shown to increase the synthesis of both the heavy and light subunits of ferritin. One could postulate that lipid peroxidation products generated by an inflammatory stimulus such as an atherogenic diet influence ferritin levels indirectly via cytokine stimulation. Another route by which lipid peroxidation products may exert indirect effects is via the nitric oxide (NO) pathway. NO synthesis has been shown to stimulate the binding of FRP to the IRE in macrophage and nonmacrophage cell lines (34). Moreover, in isolated porcine arteries, oxidized but not native LDL inhibits the production of endothelium-derived relaxing factor (NO) (23). These observations are consistent with our results in the mouse model. We observed increases in apoferritin synthesis and decreases in FRP message in mice that are resistant to fatty streak formation after $15 \mathrm{wk}$ on an atherogenic diet. Increases in the generation of lipid peroxidation products that result from feeding an atherogenic diet (10) would be expected to inhibit NO synthesis and therefore not favor the interaction of the IRE with FRP. Thus, ferritin synthesis would be allowed to increase in response to cellular demands.

We demonstrated in Fig. 2 that linear increases in extracellular iron linearly increase LDL modification. However, coincubation of cocultures with the iron chelator DETAPAC fail to decrease significantly the LDL-induced monocyte transmigration and the generation of lipid hydroperoxides. Although we do not postulate that a change in intracellular iron is the only mechanism by which lipid peroxidation can occur, we are proposing that our data provide the first evidence suggesting that 
lipid peroxidation may be an important variable in intracellular iron homeostasis.

Taken together, the in vitro and in vivo results of this study suggest that in the face of an oxidative stress, such as the feeding of a high fat atherogenic diet or by the formation of MM-LDL, the intracellular free iron level is increased. This would act to remove the inhibitory influence of FRP on apoferritin mRNA translation and thus stimulate the synthesis of new apoferritin molecules to sequester the ferrous iron and remove it from cellular reactions. As the stress is continued, a decrease in FRP synthesis would occur to ensure that apoferritin synthesis is sufficient to meet the oxidative demands put on the cell. Thus, the interplay between environmental influences (e.g., dietary iron or lipids) and genetic factors (e.g., the ability to induce apoferritin synthesis to maintain intracellular free iron homeostasis) would determine the extent of the intracellular oxidative stress and the resulting inflammatory response. The failure of the C57BL/6J mice to decrease FRP mRNA and to increase apoferritin levels could thus be a clue to their susceptibility to developing fatty streaks when on an atherogenic diet.

\section{Acknowledgments}

We thank Dr. Judith Berliner for valuable discussions; Dr. Jian-Hua Qiao for expert aortic fatty streak analysis; Greg Hough for chemotaxis assays, Thao B. Nguyen, Cynthia Harper, and Alan Wagner for excellent technical assistance; and the members of the UCLA Heart Transplant Team for collecting the aortic specimens.

This work was supported in part by U.S. Public Health Services

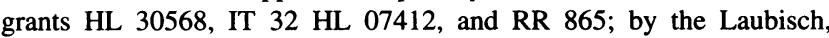
Rachel Israel Berro; by M. K. Grey Funds; and by the Cigarette and Tobacco Surtax Fund of the State of California through the TobaccoRelated Disease Research Program at the University of California.

\section{References}

1. Witztum, J. L., and D. Steinberg. 1991. Role of oxidized low density lipoprotein in atherogenesis. J. Clin. Invest. 88:1785-1792.

2. Aust, S. D., L. A. Morehouse, and C. E. Thomas. 1985. The role of transition metals in oxygen radical reactions. J. Free Radical. Biol. \& Med. 1:3-25.

3. van Eijk, H. G., and G. De jong. 1992. The physiology of iron, transferrin, and ferritin. Biol. Trace Elem. Res. 35:13-24.

4. Balla, G., H. S. Jacob, J. Balla, M. Rosenberg, K. Nath, F. Apple, J. W. Eaton, and G. M. Vercellotti. 1992. Ferritin: a cytoprotective antioxidant strategem of endothelium. J. Biol. Chem. 267:18148-18153.

5. Haile, D. J., M. W. Hentze, T. A. Rouault, J. B. Harford, and R. D. Klausner 1989. Regulation of interaction of the iron-responsive element binding protein with iron-responsive RNA elements. Mol. Cell. Biol. 9:5055-5061.

6. Rouault, T. A., M. W. Hentze, S. W. Caughman, J. B. Harford, and R. D. Klausner. 1988. Binding of a cytosolic protein to the iron-responsive element of human ferritin messenger RNA. Science (Wash. DC). 241:1207-1210.

7. Coulson, R. M. R., and D. W. Cleveland. 1993. Ferritin synthesis is controlled by iron-dependent translational derepression and by changes in synthesis/ transport of nuclear ferritin RNAs. Proc. Natl. Acad. Sci. USA. 90:7613-7617.

8. Navab, M., S. S. Imes, G. P. Hough, S. Y. Hama, L. A. Ross, R. W. Bork, A. J. Valente, J. A. Berliner, D. C. Drinkwater, H. Laks, and A. M. Fogelman. 1991. Monocyte transmigration induced by modification of LDL in cocultures of human aortic wall cells is due to induction of MCP 1 synthesis and is abolished by HDL. J. Clin. Invest. 88:2039-2046.

9. Liao, F., J. A. Berliner, M. Mehrabian, M. Navab, L. L. Demer, A. J. Lusis, and A. M. Fogelman. 1991. Minimally modified low density lipoprotein is biologically active in vivo in mice. J. Clin. Invest. 87:2253-2257.

10. Liao, F., A. Andalibi, F. C. deBeer, A. M. Fogelman, and A. J. Lusis. 1993. Genetic control of inflammatory gene induction and NF-kB-like transcription factor activation in response to an atherogenic diet in mice. J. Clin. Invest. 91:2572-2579.
11. Paigen, B., A. Morrow, C. Brandon, D. Mitchell, and P. Holmes. 1985. Variation in susceptibility to atherosclerosis among inbred strains of mice. Atherosclerosis. 57:65-73.

12. Chomczynski, P., and N. Sacchi. 1987. Single-step method of RNA isolation by acid guanidinium thiocyanate-phenol-chloroform extraction. Anal. Biochem. 162:156-159.

13. Van Lenten, B. J., and A. M. Fogelman. 1992. Lipopolysaccharide-induced inhibition of scavenger receptor expression in human monocyte-macrophages is mediated through tumor necrosis factor- $\alpha$. J. Immunol. 148:112-116.

14. Navab, M., F. Liao, G. P. Hough, L. A. Ross, B. J. Van Lenten, T. B. Rajavashisth, A. J. Lusis, H. Laks, D. C. Drinkwater, and A. M. Fogelman. 1991. Interaction of monocytes with cocultures of human aortic wall cells involves interleukins 1 and 6 with marked increases in connexin 43 message. J. Clin. Invest. 87:1763-1772.

15. Fogelman, A. M., F. Elahi, K. Sykes, B. J. Van Lenten, M. C. Territo, and J. B. Berliner. 1988. Modification of the Recalde method for the isolation of human monocytes. J. Lipid Res. 29:1243-1247.

16. Gutteridge, J. M., D. A. Rowley, and B. Halliwell. 1981. Superoxidedependent formation of hydroxyl radicals in the presence of iron salts. Detection of "free" iron in biological systems by using bleomycin-dependent degradation of DNA. Biochem. J. 199:263-265.

17. Havel, R. J., H. A. Eder, and J. H. Bragdon. 1955. The distribution and chemical composition of ultracentrifugally separated lipoproteins of human serum. J. Clin. Invest. 43:1345-1353.

18. Lowry, O. H., N. J. Rosebrough, A. L. Farr, and R. J. Randall. 1951. Protein measurement with the Folin phenol reagent. J. Biol. Chem. 193:265-275.

19. Auerbach, B. J., J. S. Kiely, and J. A. Cornicelli. 1992. A spectrophotometric microtiter-based assay for the detection of hydroperoxy derivatives of linoleic acid. Anal. Biochem. 201:375-380.

20. Navab, M., G. P. Hough, L. W. Stevenson, D. C. Drinkwater, H. Laks, and A. M. Fogelman. 1988. Monocyte migration into the subendothelial space of a coculture of adult human aortic endothelial and smooth muscle cells. J. Clin. Invest. 82:1853-1863.

21. Balla, G., G. M. Vercellotti, J. W. Eaton, and H. S. Jacob. 1990. Iron loading of endothelial cells augments oxidant damage. J. Lab. Clin. Med. 116:546-554.

22. Juckett, M. B., J. Balla, G. Balla, B. Burke, H. S. Jacob, and G. M. Vercellotti. 1993. Ferritin protects endothelial cells from oxidized low-density lipoprotein mediated cytotoxicity. Clin. Res. 41:162A.

23. Tanner, F. C., G. Noll, C. M. Boulanger, and T. F. Lüscher. 1991. Oxidized low density lipoproteins inhibit relaxations of porcine coronary arteries. Role of scavenger receptor and endothelium-derived nitric oxide. Circulation. 83:20122020.

24. Puntarulo, S., and A. I. Cederbaum. 1993. Stimulation of microsomal chemiluminescence by ferritin. Biochim. Biophys. Acta. 1157:1-8

25. Liao, F., A. Andalibi, J.-H. Qiao, H. Allayee, A. M. Fogelman, and A. J. Lusis. 1994. Genetic evidence for a common pathway mediating oxidative stress, inflammatory gene induction, and aortic fatty streak formation in mice. J. Clin. Invest. 94:877-884.

26. Bakker, G. R., and R. F. Boyer. 1986. Iron incorporation into apoferritin. The role of apoferritin as a ferroxidase. J. Biol. Chem. 261:13182-13185.

27. Coccia, E. M., V. Profita, G. Fiorucci, G. Romeo, E. Affabris, U. Testa, M. W. Hentze, and A. Battistini. 1992. Modulation of ferritin H-chain expression in Friend erythroleukemia cells: transcriptional and translational regulation by hemin. Mol. Cell. Biol. 12:3015-3022.

28. Liau, G., L. M. Chan, and P. Feng. 1991. Increased ferritin gene expression is both promoted by cAMP and a marker of growth arrest in rabbit vascular smooth muscle cells. J. Biol. Chem. 266:18819-18826.

29. Tang, C. K., J. Chin, J. B. Harford, R. D. Klausner, and T. A. Rouault. 1992. Iron regulates the activity of the iron-responsive element binding protein without changing its rate of synthesis or degradation. J. Biol. Chem. 267:2446624470.

30. Salonen, J. T., K. Nyyssonen, H. Korpela, J. Tuomilehto, R. Seppanen, and R. Salonen. 1992. High stored iron levels are associated with excess risk of myocardial infarction in eastern Finnish men. Circulation. 86:803-811.

31. Nutter, L. M., E. E. Sierra, and E. O. Ngo. 1994. Heme oxygenase does not protect human cells against oxidant stress. J. Lab. Clin. Med. 123:506-514.

32. Miller, L. L., S. C. Miller, S. V. Torti, Y. Tsuji, and F. M. Torti. 1991. Iron-independent induction of ferritin $\mathrm{H}$ chain by tumor necrosis factor. Proc. Natl. Acad. Sci. USA. 88:4946-4950.

33. Rogers, J. T., K. R. Bridges, G. P. Durmowicz, J. Glass, P. E. Auron, and H. N. Munro. 1990. Translational control during the acute phase response. Ferritin synthesis in response to interleukin-1. J. Biol. Chem. 265:14572-14578.

34. Weiss, G., B. Goossen, W. Doppler, D. Fuchs, K. Pantopoulos, G. WernerFelmayer, H. Wachter, and M. W. Hentze. 1993. Translational regulation via ironresponsive elements by the nitric oxide/NO-synthase pathway. EMBO (Eur. Mol. Biol. Org.) J. 12:3651-3657. 